\title{
Full Thermoelectric Characterization of Stoichiometric Electrodeposited Thin Film Tin Selenide (SnSe)
}

\author{
Matthew R. Burton,* Connor A. Boyle, Tianjun Liu, James McGettrick, Iris Nandhakumar, \\ Oliver Fenwick, and Matthew J. Carnie*
}

Cite This: ACS Appl. Mater. Interfaces 2020, 12, 28232-28238

Read Online

\section{ACCESS |}

Џlll Metrics \& More

回国 Article Recommendations

Supporting Information

ABSTRACT: Tin selenide ( $\mathrm{SnSe})$ has attracted much attention in the thermoelectric community since the discovery of the record figure of merit (ZT) of 2.6 in single crystal tin selenide in 2014. There have been many reports since of the thermoelectric characterization of $\mathrm{SnSe}$ synthesized or manufactured by several methods, but so far none of these have concerned the electrodeposition of SnSe. In this work, stoichiometric SnSe was successfully electrodeposited at $-0.50 \mathrm{~V}$ vs SCE as shown by EDX, XPS, UPS, and XRD. The full ZT of the electrodeposits were then measured. This was done by both a delamination technique to measure the Seebeck coefficient and electrical conductivity which showed a peak power factor of 4.2 and $5.8 \mu \mathrm{W} \mathrm{m} \mathrm{m}^{-1} \mathrm{~K}^{-2}$ for the as deposited and heat-treated films, respectively. A novel modified

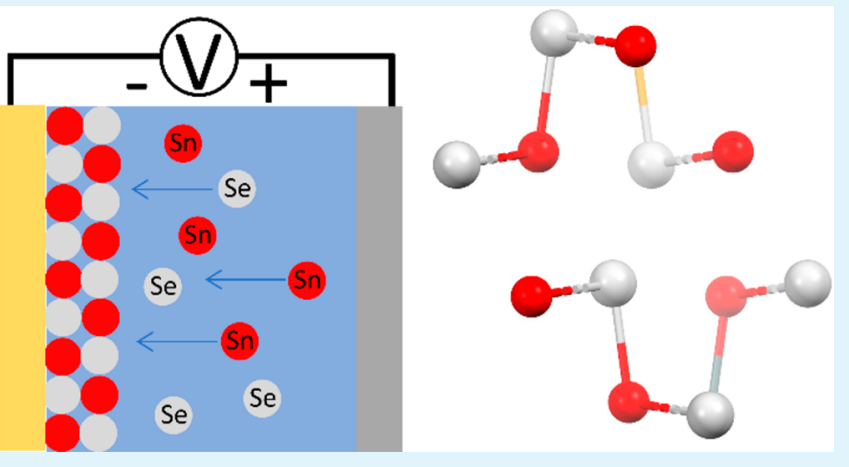
transient $3 \omega$ method was used to measure the thermal conductivity of the deposited films on the deposition substrate. This revealed the thermal conductivity to be similar to the ultralow thermal conductivity of single crystal SnSe, with a value of $0.34 \mathrm{~W} \mathrm{~m}{ }^{-1} \mathrm{~K}^{-1}$ being observed at $313 \mathrm{~K}$.

KEYWORDS: thermoelectrics, electrodeposition, tin selenide, SnSe, thin film, thermal conductivity

\section{INTRODUCTION}

The effects of climate change require greener forms of energy to be developed, while around a sixth of industrial energy is lost as heat. ${ }^{1}$ Thermoelectric generators are solid-state technologies that can harvest some of that waste heat energy. For example, thermoelectrics are being developed as a power source for self-powered devices on the Internet of things. ${ }^{2,3}$ The performance indicator that is used to compare the ability of a material to harvest the waste heat is called the figure of merit $(\mathrm{ZT})$, which is dependent on the interrelated material properties of electrical conductivity $\left(\sigma, \mathrm{S} \mathrm{m} \mathrm{m}^{-1}\right)$, Seebeck coefficient $\left(S, \mathrm{~V} \mathrm{~K}^{-1}\right)$, thermal conductivity $\left(\kappa, \mathrm{W} \mathrm{m}^{-1} \mathrm{~K}^{-1}\right)$, and the absolute temperature $(T, K)$ as shown in eq $1 .^{4}$

$$
\mathrm{ZT}=\frac{\sigma S^{2}}{\kappa} T
$$

A ZT of 1 was achieved in bismuth telluride and lead telluride in the 1950s, ${ }^{5,6}$ and ever since they have been the benchmark materials for room and medium temperature applications, respectively. Thermoelectric generators have had a degree of commercial success in space and wearable (wrist watch) ${ }^{8}$ applications, or use as Peltier coolers, ${ }^{9}$ but this has been limited. This is because until recently there has only been a modest increase in $\mathrm{ZT}$, and $\mathrm{Te}$ is a rare earth element with an abundance in the earth's crust similar to $\operatorname{Pt}\left(1 \mu \mathrm{g} \mathrm{kg}^{-1}\right) .{ }^{10}$
In 2014, Zhao et al. reported tin selenide ( $\mathrm{SnSe}$ ) to have the highest $\mathrm{ZT}$ ever recorded of 2.6 at $923 \mathrm{~K}$ along the $b$-axis. ${ }^{11} \mathrm{Of}$ note, however, is that the average $\mathrm{ZT}$ along this axis through the temperature range studied (303-973 K) was 0.95 , with the peak ZT being 2.3 and 0.84 and average ZT being 0.85 and 0.29 for the $c$-axis and $a$-axis, respectively. In fact, the authors later report the $\mathrm{ZT}$ of a device would be as low as 0.23 but highlight that through hole doping this can be increased to 1.34. This was shown to be higher than lead telluride examples. $^{12-14}$ Since 2014, SnSe has been shown to be fabricated through a variety of different techniques in the literature. These include solvothermal synthesis (SS) $)^{15-17}$ or melting (M), ${ }^{18-21}$ followed by spark plasma sintering (SPS). ${ }^{15-22}$ Other techniques include cold pressing (CP), ${ }^{23}$ hot pressing (HP), ${ }^{24,25}$ thermal evaporation, ${ }^{26,27}$ and printing. ${ }^{28}$ Many of these techniques require high temperature, high pressure, and lengthy fabrication times. In contrast, electrodeposition is a fabrication technique that is low-cost, mature,

Received: April 6, 2020

Accepted: June 1, 2020

Published: June 1, 2020

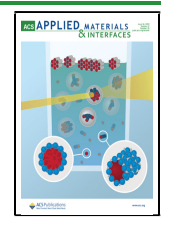


and a scalable industrial technique compatible with highthroughput continuous manufacturing. ${ }^{29,30}$ Electrodeposition of $\mathrm{SnSe}$ has been sparsely reported in the literature, ${ }^{31,32}$ and there are no reports of the thermoelectric performance of electrodeposited SnSe.

In this study we report the successful electrodeposition of thin films of SnSe. The materials properties are extensively studied through a combination of SEM, EDX, XRD, XPS, and $\mathrm{XRD}$. The full thermoelectric figure of merit $(\mathrm{ZT})$ is also measured, with the Seebeck coefficient and electrical conductivity measured through delamination and the thermal conductivity measured by the in-plane $3 \omega$ method.

\section{EXPERIMENTAL SECTION}

Thin Film Fabrication: Deionized water ( $>17.2 \mathrm{Mohm})$ was taken from a Purite (L300450); to this $\mathrm{NaOH}$ pellets (Sigma-Aldrich, 98$100.5 \%$ ) were added until a $\mathrm{pH}$ of 12 was attained. To this $\mathrm{NaOH}$ solution, $12 \mathrm{mM}$ EDTA (Sigma-Aldrich, 99\%) was added. The solution was set to stir until full dissolution of EDTA was achieved. If needed, extra $\mathrm{NaOH}$ was added to facilitate full dissolution. To $50 \mathrm{~mL}$ of this EDTA solution, $10 \mathrm{mM} \mathrm{SnCl} 2 \cdot 2 \mathrm{H}_{2} \mathrm{O}$ (Sigma-Aldrich, >98\%) was added. The solution was set to stir, while nitrogen was simultaneously gently bubbled through the solution. Once $\mathrm{Sn}$ dissolution was realized, $2.5 \mathrm{mM} \mathrm{Na}_{2} \mathrm{SeO}_{3}$ (Sigma-Aldrich, 99\%) was added to the stirring solution. Finally, $0.2 \mathrm{M} \mathrm{H}_{2} \mathrm{SO}_{4}$ was added until the $\mathrm{pH}$ of the solution was 2.5. The stirring was then halted, and after $5 \mathrm{~min}$ the nitrogen was turned off. Once the nitrogen was turned off, the deposition was started. All electrochemical experiments used an Ivium (CompactStat.e) potentiostat. The deposition was conducted via chronoamperometry, with a deposition potential of $-0.5 \mathrm{~V}$ vs SCE. The deposition time was $5400 \mathrm{~s}$. The counter electrode used was a Pt gauze electrode, with a surface area far in excess of the working electrode. The Pt gauze was cleaned in a blue flame and allowed to cool, prior to immersion into the electrolyte. The reference electrode was a commercial saturated calomel electrode (SCE) from Sentek. For all samples, except thermal conductivity samples, the working electrode composed of Si wafers (p-type (100)) with a $20 \mathrm{~nm}$ sputtered $\mathrm{Cr}$ adhesion layer, followed by a $200 \mathrm{~nm}$ sputtered Au layer. For electrical and Seebeck measurements, the electrodeposited films were delaminated onto $2 \mathrm{~mm}$ glass using 2 part epoxy as previously reported in the literature. ${ }^{33-38}$ For thermal conductivity measurements, samples were deposited onto Linseis Thin Film Analyzer "ZT chips" with a contact layer of $15 \mathrm{~nm}$ layer $\mathrm{Cr}$ evaporated onto the sample area followed by $75 \mathrm{~nm}$ of Au. Full details of the measurement process are in the Supporting Information and elsewhere. ${ }^{39,40}$ The Linseis Thin Film Analyzer measures total thermal conductance of all materials deposited onto the measurement chip plus the contribution of the chip itself, so the thermal conductivity of SnSe on its own was measured by subtracting a measurement of the chip with $\mathrm{Cr} / \mathrm{Au}$ electrode that was made before SnSe deposition. Working electrode areas were defined with Kapton tape, and $\mathrm{Cu}$ tape was used to make electrical contact between the working electrode and the potentiostat during electrodeposition.

Sample Curing: All curing of samples was performed inside an ULVAC ZEM3, as the atmosphere, cure time, and temperature could easily be controlled. The samples were laid face up inside a Ni shield and were not placed between any of the 4-point probes found inside the machine. All cure times were $30 \mathrm{~min}$ in a helium atmosphere of 1 atm, with the temperature ramped from room temperature to cure temperature in $\sim 5 \mathrm{~min}$.

Material Characterization: Sample thicknesses were measured by using a Dektak 150 surface profilometer, with all the thicknesses of all samples determined to be within $8 \pm 1 \mu \mathrm{m}$. XRD was performed on a Bruker D8 diffractor with $\mathrm{Cu} \mathrm{K} \alpha$ radiation and a 1D LYNXEYE detector. The voltage and current were $40 \mathrm{kV}$ and $40 \mathrm{~mA}$, respectively, and a step size of $0.02^{\circ}$ was used. In the BraggBrentano setup a $0.3 \mathrm{~mm}$ divergent slit was used, while in the grazing incidence setup a $2^{\circ}$ incidence angle was used with a Göebel mirror and a $0.6 \mathrm{~mm}$ slit on the primary arm. SEM and EDX were performed on a Joel 7800F field emission gun (FEG) SEM with an Oxford Laboratory EDX attachment with an accelerating voltage of $10 \mathrm{kV}$ and a working distance of $10 \mathrm{~mm}$.

X-ray photoelectron spectroscopy (XPS) and ultraviolet photoelectron spectroscopy (UPS) were performed on a Kratos Axis Supra instrument. Data were processed with CasaXPS (2.3.23rev1.1K). Samples were mounted in electrical contact with the stage to enable a $9 \mathrm{~V}$ sample bias during UPS. The Fermi edge of a Ag control, freshly cleaned by $\mathrm{Ar}^{+}$etching, was used to calibrate the energy scale to $0 \mathrm{eV}$. XPS wide scan data can be seen in Figure S3f. XPS high-resolution samples were etched to a depth of $<10 \mathrm{~nm}$ by using a monochromatic $\mathrm{Al} \mathrm{K} \alpha$ source $(225 \mathrm{~W}, 15 \mathrm{~mA}$ ) with a typical footprint of $\sim 300 \times 700$ $\mu \mathrm{m}^{2}$ and collected with a pass energy of $20 \mathrm{eV}$. Spectra were fitted with Shirley backgrounds, and synthetic components used the GL(70) line shape. As samples are electrically connected to ground, no charge correction is applied. For UPS the kinetic energy values of the secondary electron cut off and valence band maximum were determined by fitting tangents using the "step up" and "step down" "backgrounds" in CasaXPS applied to a $\mathrm{He}(\mathrm{I})(21.21 \mathrm{eV})$ photoemission spectrum collected with a $10 \mathrm{eV}$ pass energy. A typical error quoted by the instrument manufacturer is $\pm 0.13 \mathrm{eV}$.

Electrical conductivity and Seebeck coefficients were measured on a commercial ULVAC ZEM3 in a helium atmosphere; silver conductive paint (Electrolube) was added to the top and bottom to make electrical contacts. The ULVAC ZEM3 employs a 4-point probe to measure the electrical conductivity. Each Seebeck coefficient was determined by performing three differential temperature voltage measurements, taking the gradient of the results, and correcting for the internal wire Seebeck of the instrument. In-plane thermal conductivity was measured under vacuum on a test chip by a modified in-plane $3 \omega$ method with a Linseis Thin Film Analyzer (see the Supporting Information for full details).

\section{RESULTS AND DISCUSSION}

Cyclic voltammetry, as shown in Figure 1, was utilized to confirm the deposition potential for SnSe. An anodic current

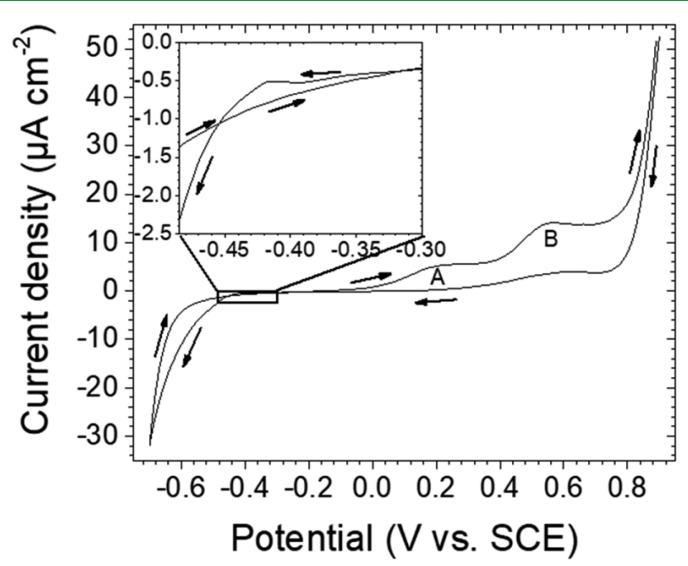

Figure 1. Cyclic voltammogram of $12 \mathrm{mM}$ EDTA, $10 \mathrm{mM} \mathrm{SnCl}_{2}$, and $2.5 \mathrm{mM} \mathrm{Na}_{2} \mathrm{SeO}_{3}$ in deionized water, acidified to a $\mathrm{pH}$ of 2.5 with $\mathrm{H}_{2} \mathrm{SO}_{4}$. The starting potential was $+0.9 \mathrm{~V}$ vs SCE, with the vertex voltage at $-0.7 \mathrm{~V}$ vs SCE before finishing at the starting voltage. The scan rate was $100 \mathrm{mV} \mathrm{s}^{-1}$.

can be seen from $-0.20 \mathrm{~V}$ vs SCE onward. The current begins to sharply increase from around $-0.4 \mathrm{~V}$ vs SCE, which is consistent with where the onset of hydrogen evolution would be seen in an aqueous electrolyte at a $\mathrm{pH}$ of 2.5. The onset of oxygen evolution is seen at just over $+0.8 \mathrm{~V}$ vs SCE, which is again consistent with a $\mathrm{pH}$ of 2.5 . On the reverse scan a nucleation loop can be observed (as highlighted in the Figure 1 
a)

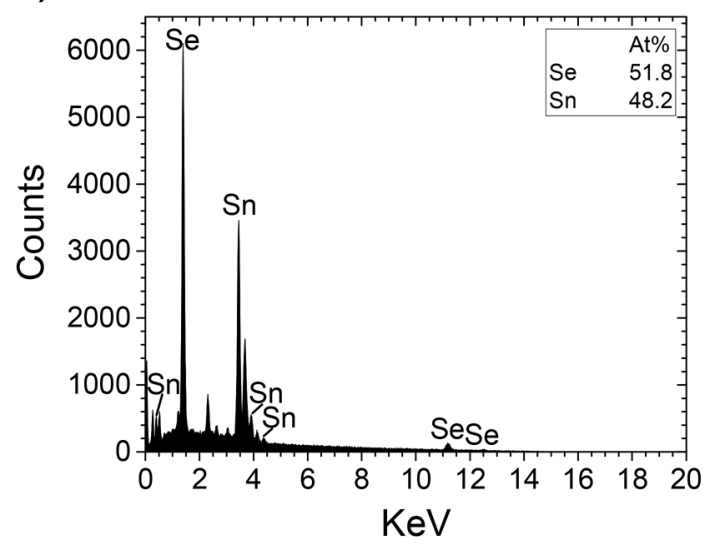

c)

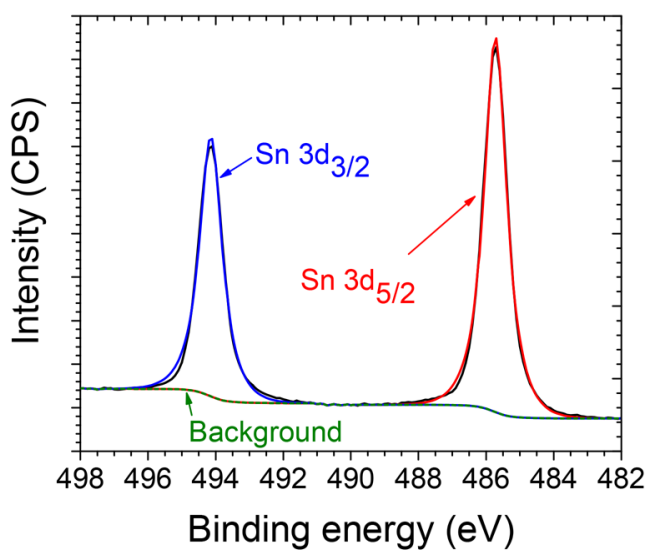

e)

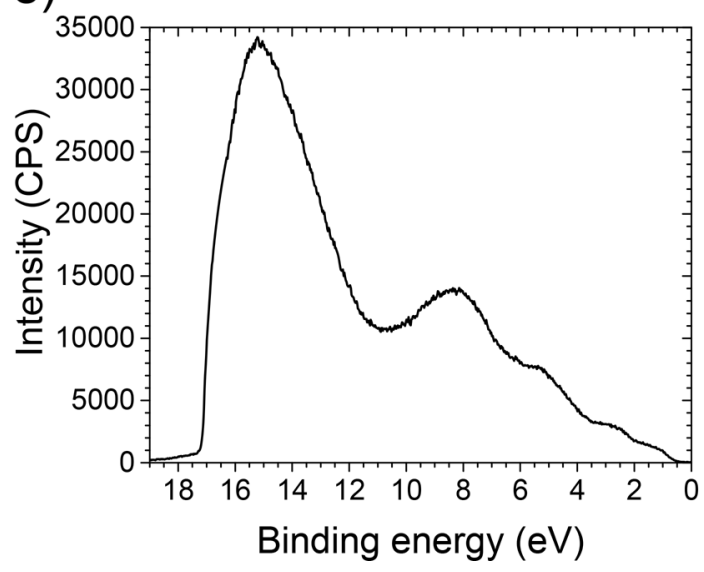

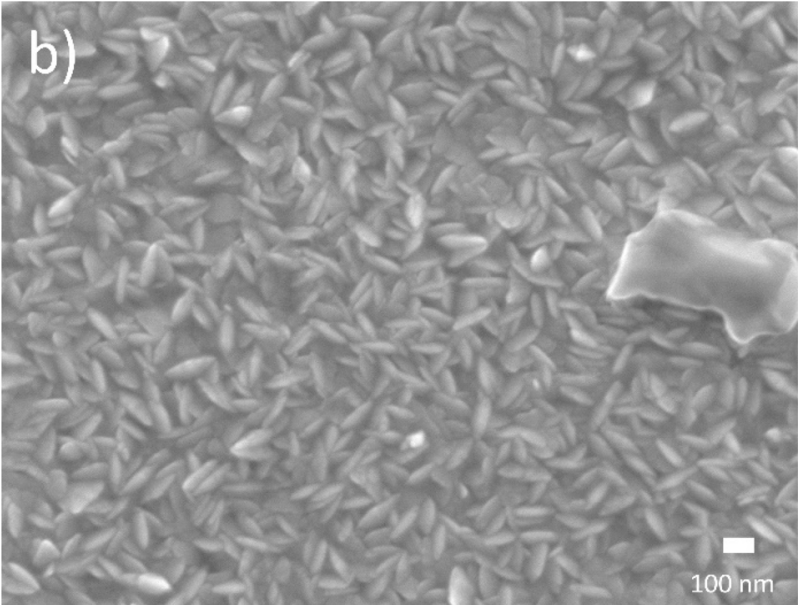

d)

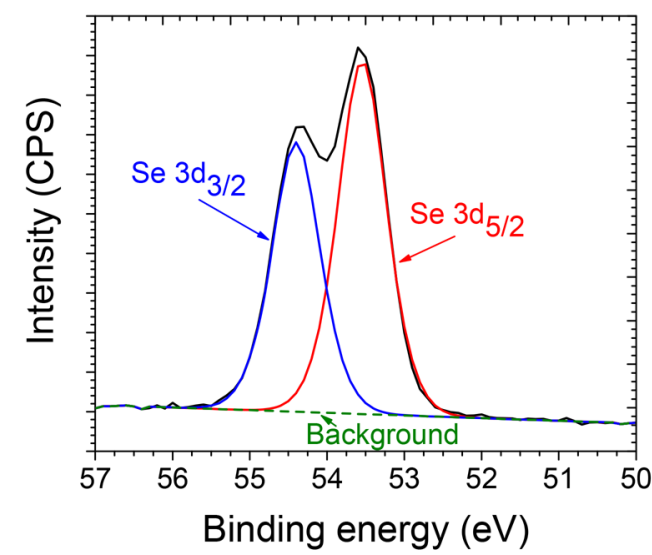

f)

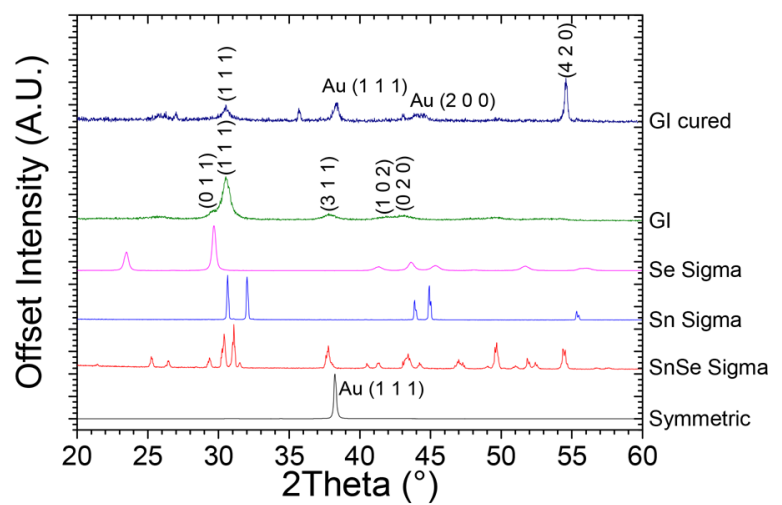

Figure 2. Material characterization of electrodeposited SnSe: (a) EDX spectrum. (b) SEM of surface morphology. (c, d) XPS showing Sn $\mathrm{n}_{3 \mathrm{~d}}$ and $\mathrm{Se}_{3 \mathrm{~d}}$ peaks, respectively. (e) UPS spectrum. (f) XRD scans of the electrodeposited film in Bragg-Brentano (symmetric) and grazing incidence (GI) setup both before and after a cure at $450 \mathrm{~K}$ in $\mathrm{He}$ and Bragg-Brentano scans of SnSe, Sn, and Se powder purchased from Sigma-Aldrich.

inset), which is indicative of a deposition having been formed on the electrode. On the forward scan two further features can be seen, which are labeled A and B in Figure 1; these correlate to the stripping of Sn and Se, respectively, as can be seen in the Supporting Information (Figure S2). A deposition potential of $-0.50 \mathrm{~V}$ vs SCE was thus determined. This was consistent with optimal results from the literature, ${ }^{32}$ where only the photoactivity of the electrodeposits was studied.

To confirm the electrodeposit was $\mathrm{SnSe}$, a variety of characterization techniques were conducted which can be seen in Figure 2. EDX revealed a bulk stoichiometry of near 1:1 $\mathrm{Sn}$ :Se ratio with a slight Se excess (Figure 2a), although this is within error of the measurement. EDX of heat-treated electrodeposited $\mathrm{SnSe}$ (Figure S3e) reveals a slight $\mathrm{Sn}$ enrichment postcuring compared to precuring, although again this is within error of the measurement. Stoichiometry measured by XPS revealed a slight $S n$ enrichment, with a $\mathrm{Sn}$ :Se ratio of 1.6:1, which is consistent with previously examined SnSe materials. ${ }^{26,28}$ The stoichiometric difference between XPS and EDX can be explained due to XPS only having a 
a)

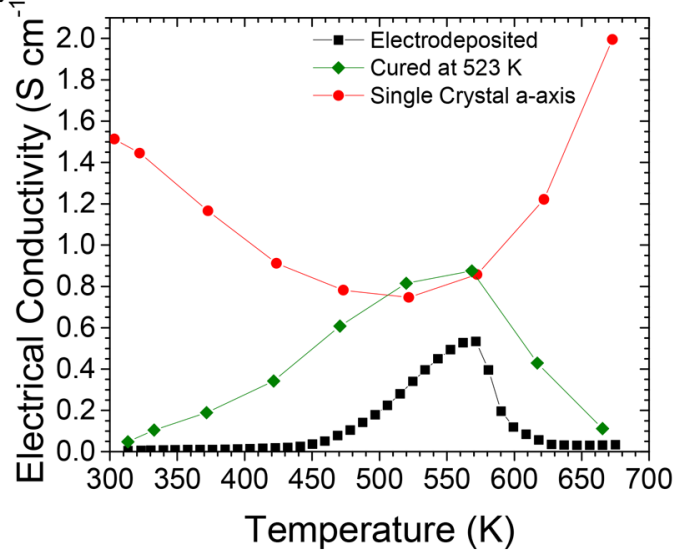

c)

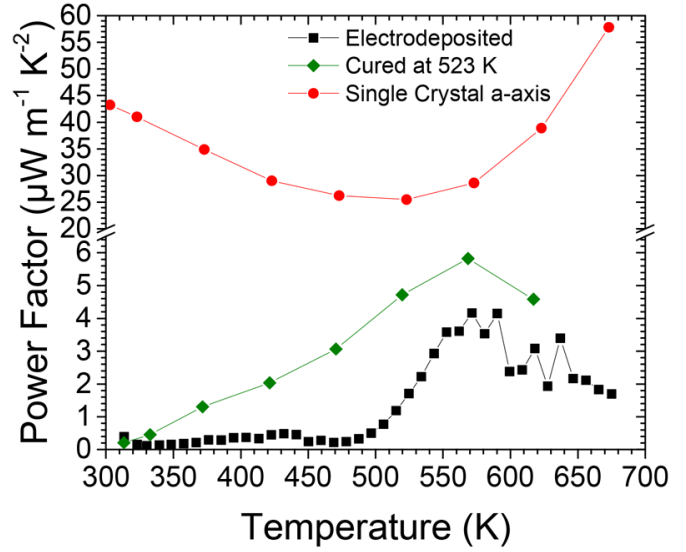

b)

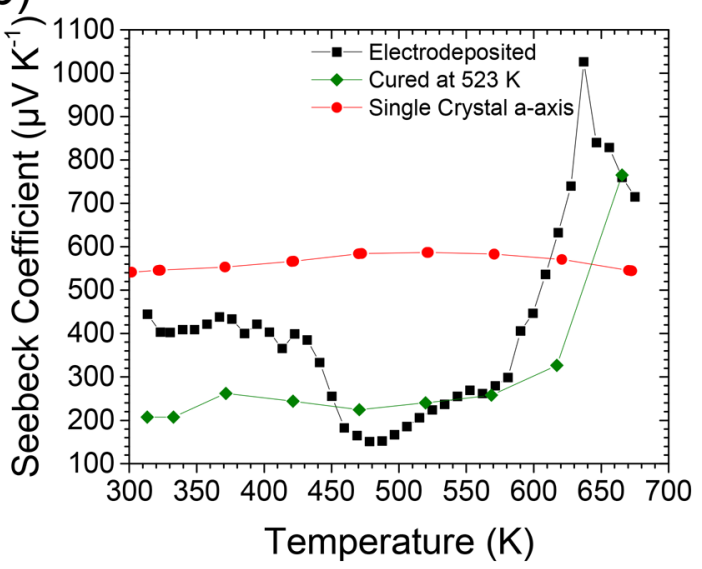

d)

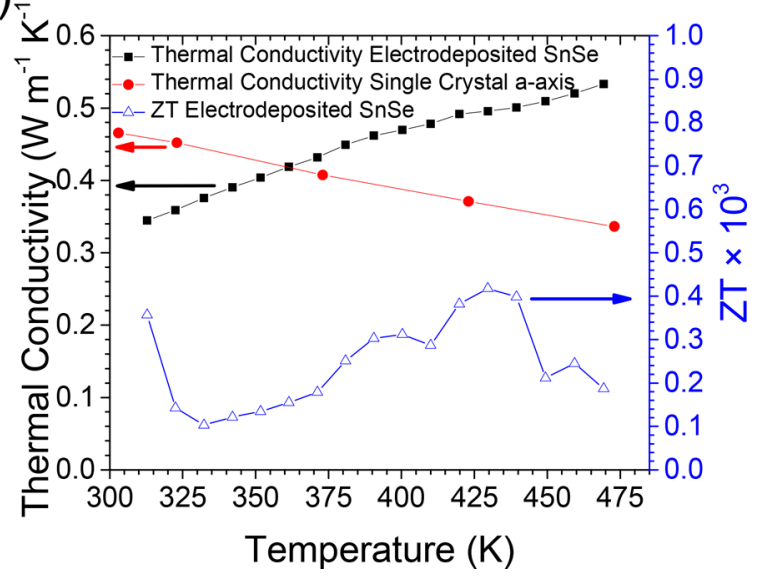

Figure 3. Thermoelectric characterization of electrodeposited SnSe both uncured and cured at $523 \mathrm{~K}$ in He for 30 min compared to the a-axis of single crystal SnSe. ${ }^{11}$ (a) Electrical conductivity, (b) Seebeck coefficient, (c) power factor, and (d) thermal conductivity and ZT.

penetration depth in the region of $10 \mathrm{~nm}$; therefore, while the bulk stoichiometry is revealed to be near ideal by EDX, XPS reveals a slight $\mathrm{Sn}$ surface enrichment. A single sharp doublet is observed for the $\mathrm{Sn}(3 \mathrm{~d}$ ) electrons (Figure $2 \mathrm{c}$ ), with the larger $3 \mathrm{~d}_{5 / 2}$ peak (FWHM $0.86 \mathrm{eV}$ ) at $485.7 \mathrm{eV}$, which is consistent with crystalline $\mathrm{SnSe}$, and notably higher than would be expected for a metallic $\mathrm{Sn}$ at $485.1 \mathrm{eV} .^{41}$ Similarly the Se 3d doublet (Figure $2 \mathrm{~d}$ ) yields a sharp Se $3 \mathrm{~d}_{5 / 2}$ peak (FWHM 0.73 $\mathrm{eV}$ ) at $53.5 \mathrm{eV}$, which is consistent with SnSe and markedly shifted from the position of $55.2 \mathrm{eV}$ expected for elemental Se. Taken together, the Sn and Se XPS peaks suggest that the electrodeposit is formed from $\mathrm{SnSe}$.

UPS (Figure 2e) revealed a kinetic energy value for the work function of the electrodeposit of $3.89 \mathrm{eV}$, which is consistent within error of density functional theory calculations of 3.91 $\mathrm{eV}$ for $\alpha$-SnSe. ${ }^{42}$ Scanning electron microscopy images (Figure S3a) reveal a porous morphology often seen for electrodeposits. Bragg-Brentano (symmetric) XRD as shown in Figure $2 \mathrm{f}$ is dominated by the underlying gold substrate; therefore, grazing incidence XRD was conducted. Grazing incidence reveals peaks that are attributed to $\mathrm{SnSe}$; however, the lack of strong peaks indicates a high level of disorder in the SnSe electrodeposit. After curing at $473 \mathrm{~K}$ for $30 \mathrm{~min}$ in a $\mathrm{He}$ atmosphere of $1 \mathrm{~atm}$, a strong additional XRD peak is revealed, suggesting a reduction in the disorder, an increase in the crystallinity, and a change of orientation. Low-resolution SEM (Figure S3b) reveals a stark change in morphology after heat treatment compared to preheat treatment (Figure S3a), while higher resolution SEM (Figure S3c and Figure 2b) reveals minimal changes in morphology. These results indicate that the large-scale porosity of the non-heat-treated films is removed through an annealing densification process, while the small-scale structure is largely unaffected.

In-plane thermoelectric characterization of the uncured and cured at $523 \mathrm{~K}$ in He electrodeposited films is shown in Figure 3. Measurements of a separate uncured electrodeposited SnSe film can be seen in Figure S4. The electrical conductivity (Figure $3 a$ ) of the uncured sample is seen to be initially significantly lower than was observed in single crystal SnSe. ${ }^{11}$ This can be explained by the high level of disorder and porosity in the electrodeposit as observed by XRD (Figure 2f). A sharp increase in the electrical conductivity is seen at $450 \mathrm{~K}$, which can be attributed to annealing of the film causing densification and an increase in the film's crystallinity. These annealing effects elevate the electrical conductivity of electrodeposited $\mathrm{SnSe}$ to $0.53 \mathrm{~S} \mathrm{~cm}^{-1}$ at $570 \mathrm{~K}$, which is near that of the $a$-axis of single crystal $\mathrm{SnSe}$ which is $0.86 \mathrm{~S} \mathrm{~cm}^{-1}$ at 572 $\mathrm{K}^{11}$ In general, though, the electrical conductivities of these films are significantly lower than that of single crystal SnSe. This can be explained from two factors. First, the films exhibit a reasonable degree of porosity, as can be seen in the lowresolution SEM in Figure S3a, which acts to reduce electrical conductivity. Second, delamination from the deposition substrate ultimately caused cracking across the film (Figure S3d). Collectively, these contributed to the lower electrical conductivity observed in these films. After curing in $\mathrm{He}$ at 523 
$\mathrm{K}$ for $30 \mathrm{~min}$, the electrical conductivity is seen to be higher compared to that of the uncured sample, throughout all temperatures observed. The electrical conductivity is briefly seen to higher than that observed in the $a$-axis of single crystal SnSe between 520 and $570 \mathrm{~K}$. The uncured films exhibit Seebeck coefficients of a similar magnitude to those seen in single crystal $\mathrm{SnSe}$ at room temperature. ${ }^{11}$ As would be expected, the Seebeck coefficient also changes sharply around $450 \mathrm{~K}$, with the drop being due to the electrical conductivity and the Seebeck coefficient having a direct and inverse correlation with carrier concentration, respectively. ${ }^{4}$ Interestingly the cured film does not exhibit the sharp change in the Seebeck coefficient around $450 \mathrm{~K}$ that was seen in the noncured film, which confirms that this is an irreversible change from curing rather than a phase change. The Seebeck coefficient of the cured film is instead seen to slightly increase with temperature, the same trend as seen in single crystal SnSe. The resulting power factors (Figure 3c) are around 10 times lower than is seen in single crystal SnSe, ${ }^{11}$ with the cured film exhibiting higher power factors throughout compared to the uncured film. The thin film SnSe power factor seen here is comparable to SnSe thin films in the literature prepared by using other methods (Figure S4d), such as thermal evaporation, ${ }^{26}$ thermal coevaporation, ${ }^{27}$ and chemical vapor deposition. ${ }^{43}$ Electrodeposition is, however, preferable to these techniques, as it is more easily scaled to thick films. ${ }^{29}$ The power factors of the electrodeposited films could not be measured beyond $675 \mathrm{~K}$ due to thermal stress on the thin films, causing excess films fractures; these fractures were clearly visible on the films post measurements. The cracking process most likely starts at just above $570 \mathrm{~K}$ due to the decline in electrical conductivity at that temperature being observed in all the samples and no fractures being visible when samples were cured at $523 \mathrm{~K}$. Nevertheless, $675 \mathrm{~K}$ is still higher than any other temperature SnSe thin films have been measured before (Figure S4d). Thermal conductivity values (Figure 3d) of the as-deposited films were measured via a modified transient $3 \omega$; these were observed to be comparable to those seen in single crystal SnSe by Zhao et al. ${ }^{11}$ Temperature and thermal conductivity are, however, in contrast to Zhao et al. seen to have a positive correlation in the temperature range 300-470 $\mathrm{K}$, which is typical of disordered films and arises from the boundaries within the films. The thermal conductivity measurement was cycled four times (Figure S5), which showed minimal changes in the thermal conductivity, although a slight increase was observed on each cycle. The boundaries along with delamination cracking and porosity ultimately explain the low ZT (Figure 3d) value observed in the film up to $470 \mathrm{~K}$. Although we expect the sharp increase in the power factor above $470 \mathrm{~K}$ to cause a similar increase in $\mathrm{ZT}$, we were unable to measure the thermal conductivity in this temperature range.

\section{CONCLUSION}

In conclusion, $\sim 8 \mu \mathrm{m}$ thick films of SnSe were fabricated onto a $\mathrm{Au}$ substrate via a simple electrodeposition process. While XPS and EDX confirmed the deposition was SnSe, XRD revealed that the as-deposited material exhibited a low level of crystallinity. This could also be seen in the positive correlation between thermal conductivity and temperature. The Seebeck coefficients of the $\mathrm{SnSe}$ electrodeposits at room temperature were similar to single crystal SnSe; however, because of the low crystallinity, porosity, and cracked nature of the delaminated films, the electrical conductivity was seen to be significantly lower below $450 \mathrm{~K}$. Remarkably, the films annealed above 450 $\mathrm{K}$, which resulted in a densification and a significant improvement in the electrical conductivity to near single crystal SnSe levels. The thermal conductivity was also measured to be comparable to that seen in single crystal SnSe. This observation represents an important step in developing thick film metal chalcogenide thermoelectrics that are deposited by scalable methods.

\section{ASSOCIATED CONTENT}

\section{Supporting Information}

The Supporting Information is available free of charge at https://pubs.acs.org/doi/10.1021/acsami.0c06026.

Detailed description of thermal conductivity measurement, CVs of Sn and Se precursors, SEM of as deposited and cured and delaminated electrodeposited SnSe films, EDX of cured film, XPS wide scan, thermoelectric comparison of 2 samples, comparison of power factor to the literature, thermal conductivity hysteresis measurement (PDF)

\section{AUTHOR INFORMATION}

\section{Corresponding Authors}

Matthew R. Burton - SPECIFIC-IKC, Materials Research Centre, College of Engineering, Swansea University, Swansea SA1 8EN, U.K.; 이이.org/0000-0002-0376-6322; Email: m.r.burton@swansea.ac.uk

Matthew J. Carnie - SPECIFIC-IKC, Materials Research Centre, College of Engineering, Swansea University, Swansea SA1 8EN, U.K.; (1) orcid.org/0000-0002-4232-1967; Email: m.j.carnie@swansea.ac.uk

\section{Authors}

Connor A. Boyle - SPECIFIC-IKC, Materials Research Centre, College of Engineering, Swansea University, Swansea SA1 8EN, U.K.

Tianjun Liu - School of Engineering and Materials Science, Queen Mary University of London, London E1 4NS, U.K.; (1) orcid.org/0000-0002-3630-0414

James McGettrick - SPECIFIC-IKC, Materials Research Centre, College of Engineering, Swansea University, Swansea SA1 8EN, U.K.

Iris Nandhakumar - School of Chemistry, University of

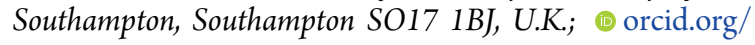
0000-0002-9668-9126

Oliver Fenwick - School of Engineering and Materials Science, Queen Mary University of London, London E1 4NS, U.K.; (1) orcid.org/0000-0001-7499-5117

Complete contact information is available at: https://pubs.acs.org/10.1021/acsami.0c06026

\section{Author Contributions}

The experiments were led by M.R.B., with M.R.B. and C.B. conducting all experiments, with the exception of thermal conductivity measurements done by T.L. and O.F., while XPS and UPS experiments were conducted by J.M. The bulk of the manuscript was written by M.R.B., with T.L. and O.F. contributing to the thermal conductivity sections and J.M. contributing to the XPS and UPS sections. All other authors proofread the manuscript and have given approval to the final version of the manuscript. 


\section{Notes}

The authors declare no competing financial interest.

\section{ACKNOWLEDGMENTS}

The authors thank EPSRC (EP/N020863/1) for funding. All authors acknowledge the SU AIM Facility (EPSRC EP/ M028267/1) for microscopy and imaging. The authors also thank COATED2 (EPSRC EP/L015099/1) for purchasing the ULVAC ZEM-3. O.F. acknowledges funding from the Royal Society (UF140372) and T.L. from the Chinese Scholarship Council. All the data created during this research are openly available from the Swansea University data archive at https:// $10.5281 /$ zenodo.3707779.

\section{ABBREVIATIONS}

$\mathrm{CV}$, cyclic voltammetry; XRD, X-ray diffraction; EDX, energydispersive X-ray spectroscopy; XPS, X-ray spectroscopy; UPS, ultraviolet photoelectron spectroscopy.

\section{REFERENCES}

(1) Besseling, J.; Pershad, H. The Potential for Recovering and Using Surplus Heat from Industry Final Report, London, 2014.

(2) Feng, R.; Tang, F.; Zhang, N.; Wang, X. Flexible, High-Power Density, Wearable Thermoelectric Nanogenerator and Self-Powered Temperature Sensor. ACS Appl. Mater. Interfaces 2019, 11 (42), 38616-38624.

(3) Park, H.; Lee, D.; Park, G.; Park, S.; Khan, S.; Kim, J.; Kim, W. Energy Harvesting Using Thermoelectricity for IoT (Internet of Things) and E-Skin Sensors. J. Phys. Energy 2019, 1 (4), 042001.

(4) Snyder, G. J.; Toberer, E. S. Complex Thermoelectric Materials. Nat. Mater. 2008, 7 (2), 105-114.

(5) Wright, D. A. Thermoelectric Properties of Bismuth Telluride and Its Alloys. Nature 1958, 181 (4612), 834-834.

(6) Joffe, A. F.; Stil'bans, L. S. Physical Problems of Thermoelectricity. Rep. Prog. Phys. 1959, 22 (1), 167.

(7) Yang, J.; Caillat, T. Thermoelectric Materials for Space and Automotive Power Generation. MRS Bull. 2006, 31 (03), 224-229.

(8) Kishi, M.; Nemoto, H.; Hamao, T.; Yamamoto, M.; Sudou, S.; Mandai, M.; Yamamoto, S. Micro Thermoelectric Modules and Their Application to Wristwatches as an Energy Source. In Eighteenth International Conference on Thermoelectrics. Proceedings, ICT'99 (Cat. No. 99TH8407); IEEE: Piscataway, NJ, 1999; pp 301-307.

(9) Rowe, D. M. CRC Handbook of Thermoelectrics; CRC Press: New York, 1995.

(10) CRC Handbook of Chemistry and Physics, 85th ed.; Lide, D. R., Ed.; CRC Press: Boca Raton, FL, 2005.

(11) Zhao, L.-D.; Lo, S.-H.; Zhang, Y.; Sun, H.; Tan, G.; Uher, C.; Wolverton, C.; Dravid, V. P.; Kanatzidis, M. G. Ultralow Thermal Conductivity and High Thermoelectric Figure of Merit in SnSe Crystals. Nature 2014, 508 (7496), 373-377.

(12) Zhao, L.-D.; Tan, G.; Hao, S.; He, J.; Pei, Y.; Chi, H.; Wang, H.; Gong, S.; Xu, H.; Dravid, V. P.; Uher, C.; Snyder, G. J.; Wolverton, C.; Kanatzidis, M. G. Ultrahigh Power Factor and Thermoelectric Performance in Hole-Doped Single-Crystal SnSe. Science (Washington, DC, U. S.) 2016, 351 (6269), 141-144.

(13) Biswas, K.; He, J.; Blum, I. D.; Wu, C.-I.; Hogan, T. P.; Seidman, D. N.; Dravid, V. P.; Kanatzidis, M. G. High-Performance Bulk Thermoelectrics with All-Scale Hierarchical Architectures. Nature 2012, 489 (7416), 414-418.

(14) Wu, H. J.; Zhao, L.-D.; Zheng, F. S.; Wu, D.; Pei, Y. L.; Tong, X.; Kanatzidis, M. G.; He, J. Q. Broad Temperature Plateau for Thermoelectric Figure of Merit ZT $>2$ in Phase-Separated PbTe0.7S0.3. Nat. Commun. 2014, 5 (1), 4515.

(15) Li, Y.; Li, F.; Dong, J.; Ge, Z.; Kang, F.; He, J.; Du, H.; Li, B.; Li, J.-F. Enhanced Mid-Temperature Thermoelectric Performance of Textured SnSe Polycrystals Made of Solvothermally Synthesized Powders. J. Mater. Chem. C 2016, 4 (10), 2047-2055.
(16) Shi, X.; Chen, Z.-G.; Liu, W.; Yang, L.; Hong, M.; Moshwan, R.; Huang, L.; Zou, J. Achieving High Figure of Merit in $\mathrm{p}$-Type Polycrystalline Sn 0.98 Se via Self-Doping and AnisotropyStrengthening. Energy Storage Mater. 2018, 10, 130-138.

(17) Shi, X.; Wu, A.; Liu, W.; Moshwan, R.; Wang, Y.; Chen, Z.-G.; Zou, J. Polycrystalline SnSe with Extraordinary Thermoelectric Property via Nanoporous Design. ACS Nano 2018, 12 (11), 11417-11425.

(18) Sassi, S.; Candolfi, C.; Vaney, J.-B.; Ohorodniichuk, V.; Masschelein, P.; Dauscher, A.; Lenoir, B. Assessment of the Thermoelectric Performance of Polycrystalline p -Type SnSe. Appl. Phys. Lett. 2014, 104 (21), 212105.

(19) Wei, T.-R.; Tan, G.; Zhang, X.; Wu, C.-F.; Li, J.-F.; Dravid, V. P.; Snyder, G. J.; Kanatzidis, M. G. Distinct Impact of Alkali-Ion Doping on Electrical Transport Properties of Thermoelectric p -Type Polycrystalline SnSe. J. Am. Chem. Soc. 2016, 138 (28), 8875-8882.

(20) Li, Y.; Shi, X.; Ren, D.; Chen, J.; Chen, L. Investigation of the Anisotropic Thermoelectric Properties of Oriented Polycrystalline SnSe. Energies 2015, 8 (7), 6275-6285.

(21) Fu, Y.; Xu, J.; Liu, G.-Q.; Yang, J.; Tan, X.; Liu, Z.; Qin, H.; Shao, H.; Jiang, H.; Liang, B.; Jiang, J. Enhanced Thermoelectric Performance in P-Type Polycrystalline SnSe Benefiting from Texture Modulation. J. Mater. Chem. C 2016, 4 (6), 1201-1207.

(22) Gong, Y.; Chang, C.; Wei, W.; Liu, J.; Xiong, W.; Chai, S.; Li, D.; Zhang, J.; Tang, G. Extremely Low Thermal Conductivity and Enhanced Thermoelectric Performance of Polycrystalline $\mathrm{SnSe}$ by $\mathrm{Cu}$ Doping. Scr. Mater. 2018, 147, 74-78.

(23) Morales Ferreiro, J. O.; Diaz-Droguett, D. E.; Celentano, D.; Reparaz, J. S.; Sotomayor Torres, C. M.; Ganguli, S.; Luo, T. Effect of the Annealing on the Power Factor of Un-Doped Cold-Pressed SnSe. Appl. Therm. Eng. 2017, 111, 1426-1432.

(24) Chen, C.-L.; Wang, H.; Chen, Y.-Y.; Day, T.; Snyder, G. J. Thermoelectric Properties of P-Type Polycrystalline SnSe Doped with Ag. J. Mater. Chem. A 2014, 2 (29), 11171.

(25) Chere, E. K.; Zhang, Q.; Dahal, K.; Cao, F.; Mao, J.; Ren, Z. Studies on Thermoelectric Figure of Merit of Na-Doped p-Type Polycrystalline SnSe. J. Mater. Chem. A 2016, 4 (5), 1848-1854.

(26) Burton, M. R.; Liu, T.; McGettrick, J.; Mehraban, S.; Baker, J.; Pockett, A.; Watson, T.; Fenwick, O.; Carnie, M. J. Thin Film Tin Selenide (SnSe) Thermoelectric Generators Exhibiting Ultralow Thermal Conductivity. Adv. Mater. 2018, 30 (31), 1801357.

(27) Jeong, G.; Jaung, Y. H.; Kim, J.; Song, J. Y.; Shin, B. Sn 1-x Se Thin Films with Low Thermal Conductivity: Role of Stoichiometric Deviation in Thermal Transport. J. Mater. Chem. C 2018, 6 (37), 10083-10087.

(28) Burton, M. R.; Mehraban, S.; Beynon, D.; McGettrick, J.; Watson, T.; Lavery, N. P.; Carnie, M. J. 3D Printed SnSe Thermoelectric Generators with High Figure of Merit. Adv. Energy Mater. 2019, 1900201.

(29) De Vogelaere, M.; Sommer, V.; Springborn, H.; MichelsenMohammadein, U. High-Speed Plating for Electronic Applications. Electrochim. Acta 2001, 47 (1-2), 109-116.

(30) Chen, H.; Wei, Z.; Zheng, X.; Yang, S. A Scalable Electrodeposition Route to the Low-Cost, Versatile and Controllable Fabrication of Perovskite Solar Cells. Nano Energy 2015, 15, 216226.

(31) Subramanian, B.; Mahalingam, T.; Sanjeeviraja, C.; Jayachandran, M.; Chockalingam, M. J. Electrodeposition of Sn, Se, SnSe and the Material Properties of SnSe Films. Thin Solid Films 1999, 357 (2), 119-124.

(32) Zainal, Z.; Hussein, M. Z.; Ali, A. J.; Kassim, A. Structure and Photoactivity of Electrodeposited Tin Selenide Films on Tin Substrate; Malaysian J. Anal. Sci. 2001, 7, 197-202.

(33) Takahashi, M.; Kojima, M.; Sato, S.; Ohnisi, N.; Nishiwaki, A.; Wakita, K.; Miyuki, T.; Ikeda, S.; Muramatsu, Y. Electric and Thermoelectric Properties of Electrodeposited Bismuth Telluride (Bi2Te3) Films. J. Appl. Phys. 2004, 96 (10), 5582-5587. 
(34) Takahashi, M.; Uosaki, K.; Kita, H.; Yamaguchi, S. Resistivity, Carrier Concentration, and Carrier Mobility of Electrochemically Deposited CdTe Films. J. Appl. Phys. 1986, 60 (6), 2046.

(35) Baranski, A. S.; Bennett, M. S.; Fawcett, W. R. The Physical Properties of CdS Thin Films Electrodeposited from Aqueous Diethylene Glycol Solutions. J. Appl. Phys. 1983, 54 (11), 6390-6394.

(36) Takahashi, M.; Muramatsu, Y.; Watanabe, M.; Wakita, K.; Miyuki, T.; Ikeda, S. Preparation and Characterization of $\mathrm{Cu}$-Doped p-CdTe Films Grown by Cathodic Electrodeposition. J. Electrochem. Soc. 2002, 149 (6), C311.

(37) Lei, C.; Ryder, K. S.; Koukharenko, E.; Burton, M.; Nandhakumar, I. S. Electrochemical Deposition of Bismuth Telluride Thick Layers onto Nickel. Electrochem. Commun. 2016, 66, 1-4.

(38) Burton, M. R; Naylor, A. J.; Nandhakumar, I. S. Electrochemically Copper-Doped Bismuth Tellurium Selenide Thin Films. Electrochem. Commun. 2018, 97, 56-59.

(39) Liu, T.; Zhao, X.; Li, J.; Liu, Z.; Liscio, F.; Milita, S.; Schroeder, B. C.; Fenwick, O. Enhanced Control of Self-Doping in Halide Perovskites for Improved Thermoelectric Performance. Nat. Commun. 2019, 10 (1), 5750.

(40) Linseis, V.; Völklein, F.; Reith, H.; Nielsch, K.; Woias, P. Advanced Platform for the In-Plane ZT Measurement of Thin Films. Rev. Sci. Instrum. 2018, 89 (1), 015110.

(41) Shalvoy, R. B.; Fisher, G. B.; Stiles, P. J. Bond Ionicity and Structural Stability of Some Average-Valence-Five Materials Studied by x-Ray Photoemission. Phys. Rev. B 1977, 15 (4), 1680-1697.

(42) Cui, Z.; Wang, X.; Ding, Y.; Li, M. Exploration Work Function and Optical Properties of Monolayer SnSe Allotropes. Superlattices Microstruct. 2018, 114, 251-258.

(43) Liu, S.; Sun, N.; Liu, M.; Sucharitakul, S.; Gao, X. P. A. Nanostructured SnSe: Synthesis, Doping, and Thermoelectric Properties. J. Appl. Phys. 2018, 123 (11), 115109. 